\title{
Uso da Gabapentina na Melhoria Sintomática da Síndrome do Túnel Cárpico: Qual a Evidência?
}

\author{
Cristina Mesquita Neves*, Adriana Vasconcelos*, Marina Carneiro*, Marta Fevereiro*
}

\section{Resumo}

Introdução: O Síndrome do Túnel Cárpico (STC) tem origem na compressão do nervo mediano do punho, causando parestesias, adormecimento e dor na mão. O tratamento da STC consiste na descompressão cirúrgica do nervo ou no tratamento conservador, nomeadamente com recurso à gabapentina.

Objetivos: O STC é, empiricamente, uma patologia muito prevalente nos cuidados de saúde primários e o médico de família depara-se com limitadas opções terapêuticas. Assim, o objetivo deste estudo é realizar uma revisão baseada na evidência acerca da eficácia do uso da gabapentina na melhoria sintomática da STC.

Métodos: Foi realizada uma pesquisa nas bases de dados Medline, Cochrane, National Guideline Clearinghouse, National Institute for Health and Care Excellence (NICE), British Medical Journal (BMJ), Database of Abstracts of Reviews of Effectiveness (DARE), Essential Evidence Plus (EEP), e principais fontes de dados portuguesas, de artigos publicados de janeiro de 2006 até novembro de 2016, utilizando as palavras-chave (termos MeSH): "gabapentin" e "carpal tunnel syndrome". Para avaliação dos níveis de evidência (NE) e atribuição de forças de recomendação (FR) foi utilizada a escala Strenght of Recommendation Taxonomy (SORT).

Resultados: Foram encontrados 37 artigos, dos quais 3 cumpriam os critérios de inclusão. 0 ensaio clínico aleatorizado (ECAC), de Hui A.C. et al., concluiu que a gabapentina não produziu redução significativa na severidade dos sintomas (NE 1). No entanto, outro ECAC e

\begin{abstract}
Introduction: The Carpal Tunnel Syndrome (CTS) originates in the compression of the median nerve of the wrist, causing paresthesia, numbness and pain in the hand. The treatment of CTS consists of surgical decompression of the nerve or conservative treatment, in particular using gabapentin.
\end{abstract}

Objectives: CTS is, empirically, a very prevalent pathology in primary health care and the family physician is faced with limited therapeutic options. Thus, the aim of this study is to conduct an evidence-based review of the efficacy of gabapentin in the symptomatic improvement of CTS.

Methods: A survey was conducted in the Medline, Cochrane, National Guideline Clearinghouse, National Institute for Health and Care Excellence (NICE), British Medical Journal (BMJ) Database of Abstracts of Reviews of Effectiveness (DARE), Essential Evidence Plus (EEP), and main sources of Portuguese data, from articles published from January 2006 to November 2016, using the keywords (MeSH terms): "gabapentin" and "carpal tunnel syndrome". The Strenght of Recommendation Taxonomy (SORT) scale was used to assess the evidence levels (EL) and assignment of strenght of recommendation (SR).

Results: They were found 37 articles, of which 3 fulfilled the inclusion criteria. The randomized controlled trial (RCT), by Hui A.C. et al., concluded that gabapentin did not produce a significant reduction in symptom severity (EL 1). However, another RCT and a prospective cohort

\footnotetext{
* USF do Mar, ACeS Póvoa de Varzim

As autoras tiveram igual contribuição para o artigo.
} 
um estudo de coorte prospetivo evidenciaram a eficácia da gabapentina na redução sintomática do STC, com NE 1 e NE 2, respetivamente.

Conclusões: Apesar da inclusão de dois ensaios clínicos de alta qualidade e NE 1, pelos achados inconsistentes entre os dois estudos e pela inclusão de uma coorte prospetiva com desenho de estudo com qualidade inferior, foi considerada não existir evidência da eficácia da gabapentina na melhoria sintomática da STC com FR B. Serão necessários mais estudos que clarifiquem a sua eficácia, mas também o esquema posológico mais adequado, efeitos adversos e benefícios a longo prazo.

Palavras-chave: gabapentina, Síndrome do Túnel Carpal

\section{Introdução}

A síndrome do túnel do cárpico (STC) é a síndrome compressiva nervosa periférica mais comum em todo o mundo. ${ }^{1}$ Esta é causada pela compressão do nervo mediano no punho, causando sintomas como parestesias, adormecimento e dor na mão, que pode irradiar para o antebraço e ombro. ${ }^{2}$ Em casos mais severos os músculos inervados pelo nervo mediano podem ser afetados e resultar na diminuição da força muscular da mão. ${ }^{1}$

A etiologia desta síndrome é ainda desconhecida, mas presume-se uma origem multifatorial. São conhecidos diversos fatores de risco como diabetes mellitus, menopausa, hipotiroidismo, obesidade, artrite e gravidez. ${ }^{1}$ A história clínica e exame objetivo permanecem como gold standard para o diagnóstico, existindo ainda bastante controvérsia acerca da necessidade de exames adicionais, como eletromiografia e ecografia, para apoiar a decisão do tratamento a efetuar. ${ }^{1}$

O tratamento do STC consiste na descompressão cirúrgica do nervo ou no tratamento conservador. Apesar de vários estudos mostrarem que o tratamento cirúrgico resulta num melhor alívio sintomático, ${ }^{2,4}$ uma meta-analise recente concluiu que a abordagem conservadora deve ser a primeira opção de tratamento do STC, reservando a abordagem cirúrgica no caso de falha da abordagem conservadora. ${ }^{3}$ Dentro das opções de tratamento conservador surgem fármacos (injeção local de corticoides, vitamina $\mathrm{B}$, diuréticos, anti-inflamatórios não esteroides e outros), laserterapia, utilização de ortóteses, terapia por ultrassons, alterações ergonómicas, fisioterapia e exercícios dirigidos. ${ }^{3}$ study evidenced the efficacy of gabapentin in reduction of CTS symptoms, with EL 1 and EL 2, respectively.

Conclusions: Despite the inclusion of two high quality and EL 1 clinical trials, the inconsistent findings between the both studies and the inclusion of a prospective cohort with lower quality study design, there was no evidence of the efficacy of gabapentin in the symptomatic improvement of CTS with SR B. Further studies will be needed to clarify its efficacy, but also the most appropriate dosing regimen, adverse effects and long-term benefits.

Keywords: gabapentin, Carpal Tunnel Syndrome

Neste contexto, alguns estudos apontam para a utilidade do uso da gabapentina no STC. ${ }^{5,6}$ A gabapentina é um medicamento antiepilético, mas é também utilizado para alívio da dor neuropática em diferentes patologias, com eficácia na neuropatia diabética, nevralgia pós-herpética e dor neuropática mista. ${ }^{7}$ Dada a componente neuropática dos sintomas do STC, é possível considerar a utilidade da gabapentina no tratamento dos casos leves a moderados do STC que são refratários a outros tratamentos conservadores ou quando a abordagem cirúrgica não é considerada. ${ }^{5}$

O STC é, empiricamente, uma patologia muito prevalente nos cuidados de saúde primários e o médico de família depara-se frequentemente com limitadas opções terapêuticas. Assim, o objetivo deste estudo é realizar uma revisão baseada na evidência acerca da eficácia do uso da gabapentina na melhoria sintomática da síndrome do túnel cárpico.

\section{Métodos}

Foi realizada uma pesquisa nas bases de dados $\mathrm{Me}$ dline, Cochrane, National Guideline Clearinghouse, National Institute for Health and Care Excellence (NICE), British Medical Journal (BMJ), Database of Abstracts of Reviews of Effectiveness (DARE), Essential Evidence Plus (EEP), e principais fontes de dados portuguesas. Foram selecionados Ensaios Clínicos (EC), MetaAnálises (MA), Revisões Sistemáticas (RS) e Normas de Orientação Clínica (NOC) publicados de janeiro de 2006 
até à data da pesquisa, em novembro de 2016. Foram admitidos na pesquisa os artigos que estivessem escritos em inglês, português e espanhol e foram utilizadas as seguintes palavras-chave (termos MeSH): "gabapentin" e "carpal tunnel syndrome".

Como critérios de inclusão consideraram-se os artigos cuja população fosse constituída por adultos $(\geq 18$ anos), com diagnóstico de Síndrome do Túnel Cárpico, e em que a intervenção fosse o uso de gabapentina comparativamente ao uso de placebo ou ausência de intervenção. 0 resultado medido foi melhoria sintomática, nomeadamente analgésica e funcional.

Foram usados como critérios de exclusão: artigos duplicados, ensaios clínicos incluídos em revisões sistemáticas, discordância com o objetivo da revisão e artigos em que a população incluísse grávidas ou pessoas previamente intervencionadas cirurgicamente para tratamento do STC.
Os artigos selecionados foram avaliados através da checklist de António Vaz Carneiro "Como avaliar a investigação clínica. O exemplo da avaliação crítica de um ensaio clínico.", tendo sido incluídos aqueles considerados de "boa qualidade". 8

Para avaliação dos níveis de evidência e atribuição de forças de recomendação foi utilizada a Escala Strength Of Recommendation Taxonomy (SORT) da American Academy of Family Physicians.

\section{Resultados}

Foram encontrados 37 artigos na pesquisa inicial, tendo sido selecionados 3 artigos (Figura 1): dois ensaios clínicos aleatorizados e controlados (ECAC) e um estudo de coorte prospetivo (Tabela 1).

Figura 1. Esquema de seleção dos artigos

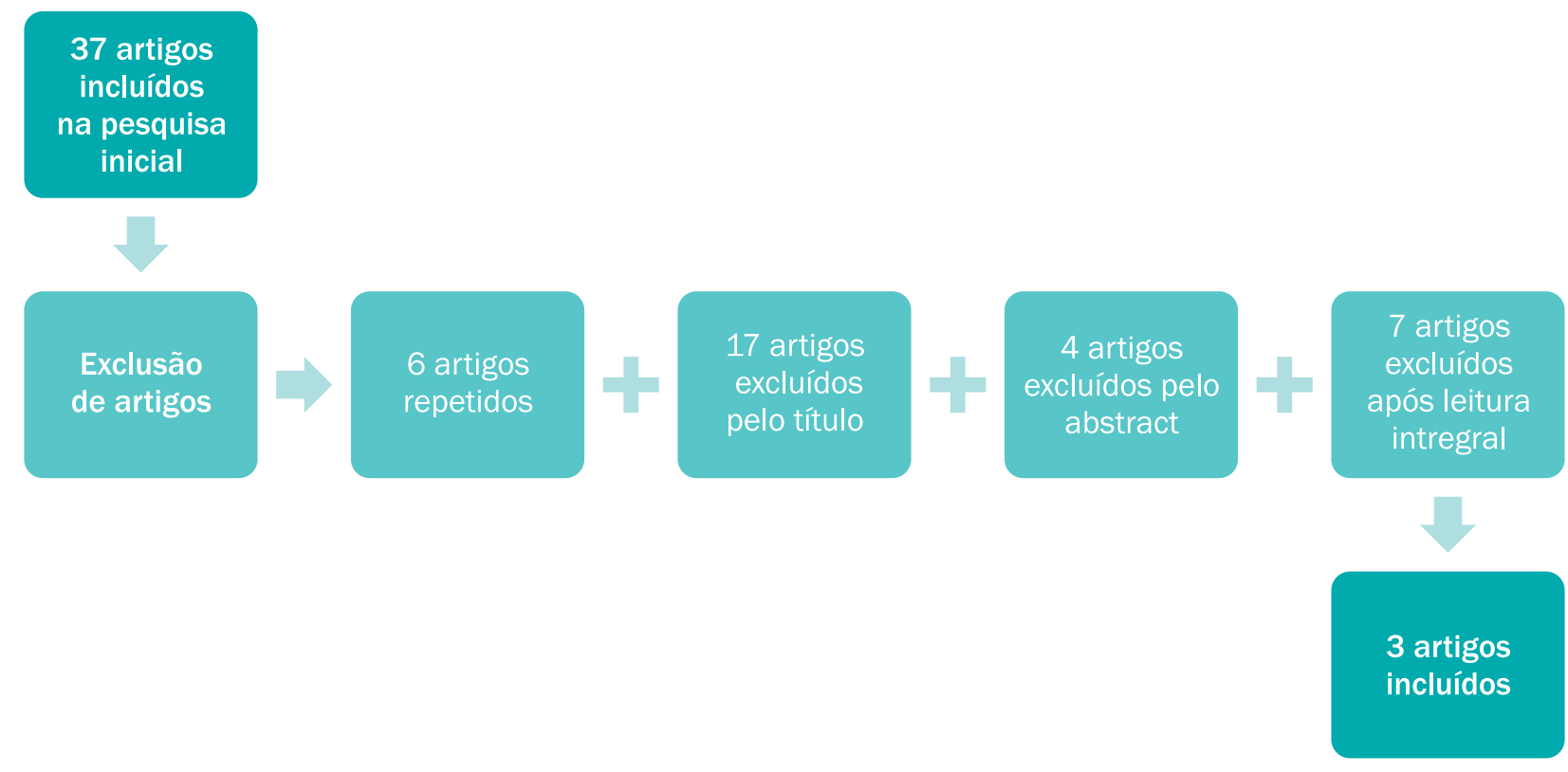

Critérios de exclusão:

- Artigos duplicados

- Ensaios clínicos incluídos em revisões sistemáticas

- Discordância com o objetivo da revisão

- População previamente intervencionada 
Tabela 1. Artigos incluídos na Revisão Baseada na Evidência

\begin{tabular}{|c|c|c|c|c|c|}
\hline REFERÊNCIA & AMOSTRA & INTERVENÇÃO & OUTCOMES & RESULTADOS & NE \\
\hline $\begin{array}{l}\text { ECAC } \\
\text { Hui A.C. et al. }{ }^{3} \\
2011\end{array}$ & $\begin{array}{l}\text { Adultos }(n=140) \text { com } \\
\text { diagnóstico de STC } \\
\text { com base clínica e } \\
\text { confirmada por EMG }\end{array}$ & $\begin{array}{l}\text { Grupo } 1 \text { - } \\
\text { gabapentina } 300 \mathrm{mg} \\
\text { id } 1 \text { semana } \rightarrow \text { bid } \\
1 \text { semana } \rightarrow \text { tid até } \\
\text { completar } 8 \text { semanas } \\
\text { Grupo } 2 \text { - placebo }\end{array}$ & $\begin{array}{l}\text { - Avaliar a melhoria } \\
\text { sintomática global } \\
\text { (GSS) às } 0,2 \mathrm{e} \\
8 \text { semanas de } \\
\text { tratamento }\end{array}$ & $\begin{array}{l}\text { - A gabapentina não produz uma } \\
\text { redução significativa na severidade } \\
\text { dos sintomas quando comparada } \\
\text { com o placebo ao longo de um } \\
\text { período de oito semanas }\end{array}$ & 1 \\
\hline $\begin{array}{l}\text { ECAC } \\
\text { Eftekharsadat } \\
\text { B. et al. }{ }^{4} \\
2015\end{array}$ & $\begin{array}{l}\text { Adultos }(n=90) \text { com } \\
\text { diagnóstico de STC } \\
\text { baseado nos critérios } \\
\text { de diagnóstico da } \\
\text { Academia Americana } \\
\text { de Neurologia }\end{array}$ & $\begin{array}{l}\text { Grupo A - } \\
\text { gabapentina } 100 \mathrm{mg} / \\
\text { dia } \\
\text { Grupo B - } \\
\text { gabapentina } 300 \mathrm{mg} / \\
\text { dia } \\
\text { Grupo C - placebo }\end{array}$ & $\begin{array}{l}\text { - Avaliar a } \\
\text { melhoria da dor } \\
\text { (VAS), melhoria } \\
\text { sintomática (SSS) } \\
\text { e funcional (FSS) } \\
\text { e a eficácia do } \\
\text { tratamento (BCTQ) } \\
\text { - Avaliar a melhoria } \\
\text { na electromiografia } \\
\text { (CMAP e SNAP) }\end{array}$ & $\begin{array}{l}\text { - Grupos A e B, em comparação } \\
\text { com o grupo C, apresentam } \\
\text { valores de VAS, SSS, FSS e BCTQ } \\
\text { significativamente menores } \\
\text { ( } p<0,05) \text {. } \\
\text { - Em todos os grupos houve melhoria } \\
\text { significativa no VAS, força de } \\
\text { pinça, SSS, FSS e score BCTQ pós } \\
\text { tratamento, exceto no CMAP e SNAP. } \\
\text { - Comparando o grupo A com o B, } \\
\text { houve melhoria significativa na } \\
\text { força de pinça e no score SSS no } \\
\text { grupo B ( } p<0,05 \text { ), mas as diferenças } \\
\text { entre os grupos noutras variáveis } \\
\text { não foram significativas. }\end{array}$ & 1 \\
\hline $\begin{array}{l}\text { Coorte } \\
\text { prospectivo } \\
\text { Taverner D. } \\
\text { et al. } .^{5} \\
2008\end{array}$ & $\begin{array}{l}\text { Adultos }(n=25) \text { com } \\
\text { STC diagnosticado por } \\
\text { EMG }\end{array}$ & $\begin{array}{l}\text { Gabapentina } 300 \mathrm{mg} \\
8-8 \mathrm{~h} \text {, com aumento } \\
\text { semanal da dose } \\
\text { até ao máximo de } \\
\text { 1,800mg/dia, ao } \\
\text { longo de } 6 \text { meses }\end{array}$ & $\begin{array}{l}\text { - Avaliar a eficácia } \\
\text { analgésica e a } \\
\text { segurança da } \\
\text { gabapentina no } \\
\text { tratamento da STC } \\
\text { - Avaliar a evolução } \\
\text { do EMG aos } \\
6 \text { meses de } \\
\text { tratamento }\end{array}$ & $\begin{array}{l}\text { - Redução estatisticamente } \\
\text { significativa da dor }(p=0,001) \text { e da } \\
\text { pontuação na escala da intensidade } \\
\text { dos sintomas ( } p=0,008) \text {, sem } \\
\text { alteração evidente na capacidade } \\
\text { funcional. } \\
\text { - Relativamente ao EMG basal, o } \\
\text { atingimento neuropático manteve- } \\
\text { se estável em } 52,6 \% \text {, melhorou } \\
\text { em } 5,3 €, \text { progrediu em } 15,8 \% \text { e } \\
\text { regrediu em } 26,3 \% \text {. }\end{array}$ & 2 \\
\hline
\end{tabular}


No ECAC de Hui A.C. et al. ${ }^{9}, 140$ adultos com diagnóstico clínico de STC e confirmado por eletromiografia (EMG), foram aleatoriamente distribuídos por dois grupos: no grupo 1 foi administrada gabapentina 300mg uma vez por dia, com aumento subsequente até três vezes por dia, durante 8 semanas, e no grupo 2 foi dado placebo. Na avaliação da melhoria sintomática global (GSS), às 0,2 e 8 semanas de tratamento, a gabapentina não produziu uma redução significativa na severidade dos sintomas quando comparada com o placebo.

Trata-se de um estudo de elevada qualidade, com dupla-ocultação e com um follow-up, pelo que se atribui um Nivel de Evidência 1.

Num outro ECAC, realizado por Eftekharsadat B. et al. ${ }^{10}$, cuja população era constituída por 90 adultos com diagnóstico de STC baseado nos critérios de diagnóstico da Academia Americana de Neurologia, a intervenção foi realizada em 3 grupos, onde variava a dose de gabapentina administrada durante dois meses (A - 100mg/ dia, B - 300mg/dia, C - placebo). Os Grupos A e B, em comparação com o grupo $\mathrm{C}$, apresentaram valores significativamente menores $(p<0,05)$ na Escala Visual Analógica (VAS), na Escala de Severidade de Sintomas (SSS), na Escala do Estado Funcional (FSS) e no Questionário de Boston (BCTQ). Após tratamento, não se verificou em nenhum grupo alterações significativas na EMG, quer a nível do componente motor quer do sensorial do potencial de ação (CMAP e SNAP, respetivamente). Comparando o grupo A e $B$, houve melhoria significativa na força de pinça e do score SSS no grupo $B(p<0,05)$, mas as diferenças entre os grupos noutras variáveis não foram significativas. 0 presente estudo concluiu que a gabapentina era útil no tratamento dos sintomas da STC, nomeadamente na dose de $300 \mathrm{mg} /$ dia.

Por se tratar de um ensaio clínico randomizado e controlado de alta qualidade, duplamente cego e com adequado follow-up, foi atribuído Nível de Evidência 1.

Em Taverner D. et al 11, um estudo de coorte prospetivo, cuja população era constituída por 25 adultos com STC diagnosticado por EMG e a intervenção era a administração de gabapentina 300mg de 8 em 8 horas, com aumento semanal da dose até à dose máxima de $1.800 \mathrm{mg} / \mathrm{dia}$, foi avaliada a eficácia analgésica e a segurança da gabapentina no tratamento da STC e avaliada a evolução do EMG aos 6 meses de tratamento.
Houve uma redução estatisticamente significativa da dor ( $p=0,001$ ) e uma redução significativa da pontuação na escala da intensidade dos sintomas ( $p=0,008)$, sem alteração evidente na capacidade funcional. Relativamente ao EMG basal, o atingimento neuropático manteve-se estável em 52,6\% dos casos, melhorou em 5,3\%, progrediu em $15,8 \%$ e em $26,3 \%$ a EMG foi normal.

As principais limitações deste estudo encontradas pelas autoras foram o baixo número de participantes $(n=25)$ e um follow-up de $76 \%$, pelo que se atribuiu um Nível de Evidência 2.

\section{Conclusões}

As autoras consideram que apesar de terem sido incluídos como evidência dois ensaios clínicos de alta qualidade e com Nível de Evidência 1, pelos achados inconsistentes entre os dois estudos e pela inclusão de uma coorte prospetiva com desenho de estudo com qualidade inferior, foi considerada não existir evidência da eficácia da gabapentina na melhoria sintomática da STC com força de recomendação $B$.

Apesar de em dois dos estudos se ter verificado alguma melhoria sintomática do STC, deve ser considerada a existência de algumas limitações, pois os estudos apresentam amostras com dimensões distintas, seguidas por curtos e diferentes intervalos de tempo (2 a 6 meses). 0 curto período de seguimento não permite antever efeitos a longo prazo ou a duração-alvo da utilização da gabapentina para obtenção dos efeitos pretendidos. Além disso, utilizaram-se diferentes posologias (100$1800 \mathrm{mg} / \mathrm{dia})$, sendo que nem todas apresentavam a mesma eficácia.

Desta forma, no futuro serão necessários novos estudos, multicêntricos, com amostras maiores e um período de seguimento mais longo, que clarifiquem: a eficácia da gabapentina, o esquema posológico, a duração-alvo da administração da gabapentina, os efeitos laterais exatos da gabapentina nas diferentes doses, a diferença do benefício da gabapentina usada isoladamente ou como complemento do tratamento cirúrgico, os benefícios a longo prazo e a população-alvo com maior benefício deste tratamentos (tendo em conta a idade, duração e intensidade/gravidade dos sintomas). 


\section{Referências Bibliográficas}

1. Padua L, Coraci D, Erra C, et al. Carpal tunnel syndrome: clinical features, diagnosis, and management. The Lancet Neurology 2016;15(12):1273-1284.

2. Verdugo R, Salinas R, Castillo J, Cea G. Surgical versus nonsurgical treatment for carpal tunnel syndrome. Cochrane Database of Systematic Reviews 2008.

3. Ren Y, Wang X, Wei Z, et al. Efficacy, safety, and cost of surgical versus nonsurgical treatment for carpal tunnel syndrome. Medicine 2016;95(40):e4857.

4. Shi Q, MacDermid J. Is surgical intervention more effective than non-surgical treatment for carpal tunnel syndrome? a systematic review. J Orthop Surg Res 2011;6(1):17.

5. Duman I, Aydemir K, Ozgul A, Kalyon T. Assessment of the Efficacy of Gabapentin in Carpal Tunnel Syndrome. J Clin Rheumatol 2008;14(3):175-177.

6. Erdemoglu A. The efficacy and safety of gabapentin in carpal tunnel patients: Open label trial. Neurol India 2009;57(3):300.

7. Moore R, Wiffen P, Derry S, Rice A. Gabapentin for chronic neuropathic pain and fibromyalgia in adults. Cochrane Database of Systematic Reviews. 2014.

8. Carneiro, A.V. "Como avaliar a investigação clínica. o exemplo da avaliação crítica de um ensaio clínico." GE- J Port Gastrenterol 2008; 15: 30-36

9. Hui AC, Wong SM, Leung HW, Man BL, Yu E, Wong LK. Gabapentin for the treatment of carpal tunnel syndrome: a randomized controlled trial. Eur.J Neurol 2011/5; 5: 726-730.

10. Eftekharsadata B, Babaei-Ghazanib A and Habibzadehc A. The Efficacy of 100 and $300 \mathrm{mg}$ Gabapentin in the Treatment of Carpal Tunnel Syndrome. Iran J Pharm Res 2015; 14 (4): 1275-1280.

11. Taverner D, Lisbona MP, Segalés N, et al. Eficacia de la gabapentina en el tratamiento del sindrome del tunel carpiano; Med Clin (Barc) 2008;130(10):371-3.

\section{Conflito de Interesses}

As autoras declaram não ter conflitos de interesses. 